\title{
Disruption by speech of serial short-term memory: The role of changing-state vowels
}

\author{
ROBERT W. HUGHES \\ Cardiff University, Cardiff, Wales \\ SÉBASTIEN TREMBLAY \\ Université Laval, Quebec City, Quebec, Canada \\ and \\ DYLAN M. JONES \\ Cardiff University, Cardiff, Wales
}

\begin{abstract}
Serial short-term memory is markedly impaired by the presence of irrelevant speech so long as the successive tokens within the irrelevant speech are phonologically (or acoustically) dissimilar (Jones $\&$ Macken, 1995b). In two experiments in which consonant-vowel-consonant syllables were used as irrelevant speech tokens, we sought to evaluate the relative disruptive potency of changes in the final consonant only (Experiment 1), in the initial consonant, or in the vowel portion (Experiment 2) of each token. The results suggest that the vowel changes are the dominant source of disruption. This dominance may be explained, at least in part, by the role played by vowel sounds in the perceptual organization of speech and, in turn, the particular propensity for vowel changes to yield information about serial order. The results are consistent also with the view that the factors that promote order encoding in sound are also the ones that promote disruption.
\end{abstract}

In a seminal study, Colle and Welsh (1976) found that background speech disrupted serial recall of visually presented items, despite the fact that the participants had been instructed to ignore the speech. This so-called irrelevant sound effect has been widely and frequently replicated and has contributed to the understanding of the interrelations between perception, attention, and memory (for recent discussions, see, e.g., Baddeley, 2000; Jones \& Tremblay, 2000; Neath, 2000). In the present study, we investigated a phenomenon first reported by Jones and Macken (1995b) — namely, that a sequence of irrelevant speech tokens that are phonologically dissimilar from each other causes more disruption than does a sequence of phonologically similar tokens. Using consonant-vowelconsonant (CVC) syllables, we examined whether any kind of phonological change between successive speech tokens gives rise to a phonological dissimilarity effect or whether some attributes (e.g., vowels, as compared with consonants) assume more importance than others.

Generally, many aspects of the irrelevant sound effect command a good degree of consensus: The intensity of the sound is not an influential variable, at least within the

This research was supported by the U.K.'s Economic and Social Research Council and by a grant from the Fonds de recherche sur la nature et les technologies du Québec. Thanks are due Bill Macken for a critical reading of an earlier version of this article. Correspondence may be addressed to R. W. Hughes, School of Psychology, Cardiff University, Cardiff CF10 3AT, Wales (e-mail: hughesrw@cardiff.ac.uk). range of 40-76 dB(A) (Colle, 1980; see also Tremblay \& Jones, 1999); the effect occurs within memory, not at encoding (Miles, Jones, \& Madden, 1991); the meaning of sound (when speech is used) plays little if any role in the effect (Buchner, Irmen, \& Erdfelder, 1996); and tasks that rely heavily on, or encourage, a serial rehearsal strategy (e.g., serial recall) are particularly susceptible to disruption (e.g., Jones \& Macken, 1993).

An early account of the irrelevant sound effect proposed that the effect is confined to speech and, more specifically, that the effect is a function of the degree of phonological similarity between the irrelevant material and the to-beremembered items (Salamé \& Baddeley, 1982). However, several lines of evidence now converge to refute this account, including the finding that irrelevant nonspeech sounds (e.g., tones) can disrupt serial recall (e.g., Jones \& Macken, 1993). Moreover, a study by Jones and Macken (1995b) provided more direct evidence against the phonological interference account by demonstrating that the degree of phonological similarity between the relevant and the irrelevant material was not, in fact, a strong predictor of the level of disruption (see also Larsen, Baddeley, \& Andrade, 2000; LeCompte \& Shaibe, 1997).

The study of Jones and Macken (1995b; Experiments 3 and 4) did reveal, however, that the degree of phonological dissimilarity within the irrelevant sequence does strongly dictate the degree of disruption. Specifically, an irrelevant sequence made up of the nonrhyming words hat, cow, nest . . . or deaf, pay, bell ... was more disruptive than a sequence made up of the rhyming words sea, flea, key... 
or door, war, more ... In a more recent study, Larsen et al. (2000) replicated this within-irrelevant-stream phonological dissimilarity effect in some of their conditions, but not in others. Their failure to observe consistently a phonological dissimilarity effect is likely to be due to the fact that they presented only one speech token concurrently with each of the six to-be-remembered items. This token dose- the number of irrelevant tokens presented on a given trial (see Bridges \& Jones, 1996) - of only 6 tokens is far smaller than the token dose of 34 tokens used by Jones and Macken (1995b). In light of the fact that disruption is known to increase as a function of token dose (Bridges \& Jones, 1996), a token dose of 6 may have rendered the study of Larsen et al. (2000) somewhat insensitive to a phonological dissimilarity effect.

The phonological dissimilarity effect is consistent with the so-called changing-state hypothesis, according to which disruption will be a function of the degree to which the irrelevant sequence exhibits acoustical change between its successive elements (Jones, Madden, \& Miles, 1992). Thus, according to the changing-state hypothesis, the effect of within-stream phonological dissimilarity constitutes a manifestation of the more general acoustic-based changing-state effect (Jones \& Macken, 1995b). ${ }^{1}$ However, a limitation shared by the studies of Jones and Macken (1995b) and Larsen et al. (2000) is that phonological dissimilarity was always operationalized purely in terms of the rime or vowel portion of the speech. Moreover, in neither study was there always a strict control of (1) whether or not there were changes in the consonant sounds across the tokens within either the phonologically similar or the phonologically dissimilar condition, (2) whether or not there were changes in the identity of consonants across the various irrelevant speech conditions, or (3) the overall structure of the tokens (e.g., CV vs. VC), either within or across the various irrelevant speech conditions.

The aim of the present study was to begin examining which of the several characteristics of an acoustically complex stimulus such as a speech token play a role in the phonological dissimilarity effect. We therefore focused on syllables with a CVC structure and sought to isolate the contribution to the phonological dissimilarity effect of a change only in the final consonant, a change only in the initial consonant, and a change only in the vowel.

\section{EXPERIMENT 1}

The goals of Experiment 1 were to (1) replicate the within-stream phonological dissimilarity effect (Jones $\&$ Macken, 1995b) and (2) to examine for the first time whether having a sequence in which only a single consonant changes from one token to the next (the final consonant in each of a sequence of CVC syllables) is sufficient to produce the effect.

\section{Method}

Participants. Twenty-six Cardiff University undergraduates participated in exchange for course credit. All were native English speakers, and all reported normal hearing and normal or correctedto-normal vision.

Materials. Nine to-be-remembered items were presented sequentially on the screen of an Apple Macintosh at a rate of one per second. The sequences were constructed from random orderings of nine consonants - $f, k, l, m, q, r, s, t$, and $v$-with the constraint that a consonant was not presented in the same serial position in two consecutive lists.

The auditory items were produced by a male native English speaker. They were digitized at a $22.5 \mathrm{kHz}$ sampling rate and to a resolution of 8 bits, using the Sound Designer II software. Three irrelevant speech sequences were constructed. Each sequence had a token dose (see Bridges \& Jones, 1996) of 24 items, consisting of 12 CVC monosyllables presented twice (see the Appendix for a list of the 12 stimuli used). In one condition, the all-components-changing condition, each component of each CVC syllable (i.e., initial consonant, vowel, and final consonant) differed from one item to the next. In a second condition, the final-consonant-only-changing condition, only the final consonant of each of the 12 CVCs changed from one to the next. In a third condition, the steady-state condition, a single item, chosen randomly from the set of $12 \mathrm{CVCs}$ for each trial, was repeated 24 times. Each item lasted approximately $300 \mathrm{msec}( \pm 20 \mathrm{msec})$ and was separated from the next by a $500-\mathrm{msec}$ silent gap. In each condition, the irrelevant speech sequence was presented throughout the to-be-remembered list, plus a $10 \mathrm{sec}$ interval between the last item and the recall cue. The irrelevant speech was presented at approximately $65 \mathrm{~dB}(\mathrm{~A})$. A quiet control condition was also included.

Design. A repeated measures design was used in which there were two independent variables: serial position (nine levels) and auditory condition (four levels). There were 15 trials per auditory condition, making 60 trials in all. The auditory conditions were presented pseudorandomly, with the constraint that each condition was represented once every 4 trials.

Procedure. The participants were tested individually in a soundproof laboratory and were seated approximately $0.5 \mathrm{~m}$ from the screen. At the outset, the participants were given standard instructions on the computer screen, informing them of the requirements for serial recall and instructing them to ignore any sounds they might hear. The participants were also informed that the sounds would not contain any messages and that they would not be tested on their contents. The experimental trials were preceded by a short practice session of three trials without irrelevant speech. Each trial was initiated by the participant, using a mouse to click a SuperCard button on the screen. This initiated the presentation of the stimulus sequence, in which the consonants were displayed individually at a rate of one per second (on for $800 \mathrm{msec}$, off for $200 \mathrm{msec}$ ). When nine consonants had been presented, the word wait flashed for $10 \mathrm{sec}$, during which the participants were expected to rehearse covertly. The word recall was then displayed to prompt the participant to attempt to write down the consonants in strict serial order. The irrelevant sound was played over headphones throughout the presentation and rehearsal phases and was terminated when the recall cue appeared. The experiment lasted some $45 \mathrm{~min}$.

\section{Results and Discussion}

Performance was scored on the basis of a strict serial order criterion: An item was scored as correct only if it corresponded to its presentation position. Table 1 shows the mean proportion of items correctly recalled in each of the four auditory conditions, pooled across serial positions.

A 4 (auditory condition) $\times 9$ (serial position) repeated measures ANOVA showed a main effect of auditory condition $\left[F(3,75)=16.01, M S_{\mathrm{e}}=8.31, p<.0001\right]$ and a main effect of serial position $\left[F(8,200)=50.40, M S_{\mathrm{e}}=\right.$ $10.48, p<.0001]$. There was no significant interaction be- 
Table 1

Means and Standard Errors for the Proportion of Items Correctly Recalled in the Quiet, Steady-State, Final-ConsonantOnly-Changing, and All-Components-Changing Conditions, Pooled Over Serial Positions in Experiment 1

\begin{tabular}{lcc}
\hline \multicolumn{1}{c}{ Condition } & $\begin{array}{c}\text { Mean Proportion } \\
\text { Correct }\end{array}$ & $\begin{array}{c}\text { Standard } \\
\text { Error }\end{array}$ \\
\hline Quiet & .58 & .014 \\
Steady state & .52 & .015 \\
Final consonant changing & .50 & .015 \\
All components changing & .46 & .015 \\
\hline
\end{tabular}

tween serial position and auditory condition $[F(24,600)=$ $\left.1.35, M S_{\mathrm{e}}=2.40, p>.05\right]$. Planned comparisons showed that recall in the all-components-changing condition was significantly poorer than that in either the final-consonantonly-changing condition $[F(1,75)=5.71, p<.05]$ or the steady-state condition $[F(1,75)=13.85, p<.001]$. However, there was no significant difference between the steady-state condition and the final-consonant-onlychanging condition $[F(1,75)=1.77, p>.05]$. Moreover, the present experiment revealed a reliable degree of disruption in the steady-state condition, as compared with the quiet condition $[F(1,75)=9.39, p<.05]$ as has sometimes (e.g., LeCompte, 1995), but not always (LeCompte, 1996), been found in previous studies.

The present experiment yielded a compelling withinstream phonological dissimilarity effect, thereby replicating a finding first reported by Jones and Macken (1995b): When successive irrelevant syllables did not have any phonemes in common (all-components-changing condition), there was more disruption than when the same syllable was repeated or when only one phoneme (the final consonant) changed from one syllable to the next. Interestingly, the fact that a sequence in which the final consonant changed from one token to the next did not cause reliably more disruption than did the steady-state condition suggests that the disruptive power of the all-componentschanging sequence stemmed from either the change of initial consonant or the change in vowel (or both). In Experiment 2, we sought to tease apart the contributions of initial-consonant change and vowel change to the phonological dissimilarity effect obtained in Experiment 1.

\section{EXPERIMENT 2}

In this experiment, within-stream phonological dissimilarity was manipulated by contrasting the effect of two sequences again consisting of CVC syllables. In one sequence, successive syllables differed only by their initial consonant, whereas in another sequence, they differed only by the vowel.

\section{Method}

Participants. Twenty-five students from Cardiff University, each a native English speaker, volunteered to participate in exchange for course credit. All the participants reported normal hearing and normal or corrected-to-normal vision.

Apparatus and Materials. The apparatus and the to-beremembered lists were the same as those in Experiment 1. Three new irrelevant speech sequences were created: a sequence in which only the vowel changed from one token to the next (vowel-onlychanging condition; /bæb/, /brb/, /bob/, and /bub/, looped in this fixed order), a sequence in which only the initial consonant changed between successive syllables (initial-consonant-only-changing condition; /dæb/, /kæb/, /pæb/, and /tæb/, looped in this fixed order), and a steady-state sequence in which the syllable /bæb/ was repeated. The three sequences were stored as sound resources within the SuperCard 2.5 software. All the tokens lasted approximately $250 \mathrm{msec}$, and the length of the silent gap between successive tokens was $50 \mathrm{msec}$. Thus, the rate of presentation was 3 tokens per second and so the token dose in this experiment was 57. In addition to the three irrelevant speech conditions, a quiet control condition was included.

Design. A repeated measures design was used in which the ordering of trials was prearranged randomly, with the constraint that each of the four conditions was represented once every 4 trials. The participants received 15 trials in each condition, making 60 trials in all.

Procedure. The procedure was the same as that in Experiment 1.

\section{Results and Discussion}

Performance was assessed according to a strict serial recall criterion, as in Experiment 1 . Table 2 shows the mean proportion of items correctly recalled in each of the four auditory conditions, pooled across serial positions. It is noticeable, first, that overall performance was higher in this experiment than in Experiment 1. Other than being due to a sampling error, it is unclear why this was the case.

A 4 (auditory condition) $\times 9$ (serial position) repeated measures ANOVA revealed a main effect of auditory condition $\left[F(3,87)=9.55, M S_{\mathrm{e}}=3.68, p<.001\right]$ and serial position $\left[F(8,232)=28.43, M S_{\mathrm{e}}=5.36, p<.0001\right]$. The interaction between serial position and auditory condition was not significant $\left[F(24,696)=1.84, M S_{\mathrm{e}}=0.91, p>\right.$ $.05]$.

Planned comparisons showed that there was no reliable difference between the steady-state condition and the initial-consonant-only-changing condition $[F(1,87)=$ $2.07, p>.05]$. Of particular importance, the vowel-onlychanging condition produced more disruption of serial recall than did both the initial-consonant-only-changing condition $[F(1,87)=4.01, p<.05]$ and the steady-state condition $[F(1,87)=10.81, p<.05]$. Again, the steady-state condition produced reliably more disruption than did the quiet condition $[F(1,87)=3.01, p=.05]$. In sum, Experiment 2 demonstrated that, for $\mathrm{CVC}$ tokens at least, when the vowel changes between successive tokens, there is a clear phonological dissimilarity effect, whereas if only the

Table 2

Means and Standard Errors for the Proportion of Items Correctly Recalled in the Quiet, Steady-State, Initial Consonant-Only-Changing, and Vowel-Only-Changing Conditions, Pooled Over Serial Positions in Experiment 2

\begin{tabular}{lcc}
\hline \multicolumn{1}{c}{ Condition } & $\begin{array}{c}\text { Mean Proportion } \\
\text { Correct }\end{array}$ & $\begin{array}{c}\text { Standard } \\
\text { Error }\end{array}$ \\
\hline Quiet & .69 & .034 \\
Steady state & .62 & .028 \\
Initial consonant changing & .63 & .027 \\
Vowel changing & .58 & .030 \\
\hline
\end{tabular}


initial consonant changes, there is no reliable disruption over and above that produced by a steady-state sequence.

Before turning to consider the implications of the findings, some mention should perhaps be made of the reliable disruption found with the steady-state, relative to the quiet control, condition in both experiments. Although this effect was relatively robust in the present study, we feel that it would still be premature to attempt to assign any functional significance to it, because a steady-state effect is sometimes found to be reliable (e.g., LeCompte, 1995) and other times not (e.g., LeCompte, 1996). However, the changing-state effect - the disruption produced by a changing-state, as compared with a steady-state, sequence - is highly robust (but for a discussion of possible reasons why a steady-state effect is sometimes observed, see Jones, Saint-Aubin, \& Tremblay, 1999).

\section{GENERAL DISCUSSION}

The present results replicate an observation first made by Jones and Macken (1995b) — namely, that a phonologically dissimilar sequence of speech tokens produces more disruption to serial recall than does a phonologically similar sequence. The results refine our understanding of the phonological dissimilarity (or changing-state irrelevant speech) effect by establishing that consonant changes - at least when located at the beginnings and endings of speech tokens - are impotent in terms of producing disruption, relative to a steady-state condition, and that a vowel must be changing from one token to the next. The fact that we used a token dose of 24 and 57 in Experiments 1 and 2, respectively, and obtained reliable phonological dissimilarity effects also indirectly supports our suggestion that Larsen et al. (2000) failed, in some conditions, to observe such effects because they used a token dose of only 6 . A direct test of this is beyond the scope of the present article but is clearly an issue that is worthy of further investigation.

A plausible explanation of the results can be offered from within an interference-by-process approach to the irrelevant sound effect (e.g., Jones \& Tremblay, 2000). On this account, disruption by irrelevant sound is a function of the degree to which it automatically yields information about the order of its constituent elements. This obligatorily and preattentively encoded order information is thought to conflict with the process of retaining the order of the to-be-remembered items. It is well established that for an auditory sequence to yield order cues, it must exhibit acoustic change between its constituent elements and, critically, these acoustic changes must constitute variations on a common ground (Bregman, 1990; Jones, Alford, Bridges, Tremblay, \& Macken, 1999). That change on a common ground is a key principle in the irrelevant sound effect was first shown by Jones and Macken (1995a). They found that if a sequence of changingstate tokens $(x, j, u, x, j, u \ldots)$ was presented such that the changes crossed different spatial locations ( $x$ always presented to the left ear, $j$ always presented to both ears, and $u$ always presented to the right ear), the disruption was markedly attenuated (relative to a steady-state condi- tion), as compared with cases in which all the tokens were presented binaurally.

The change-on-a-common-ground principle may provide a means of accounting for the difference between the effects of vowel changes and consonant changes obtained in the present study. It has been suggested that, in speech, the common ground - which allows the perceptual system to integrate, over time, different speech utterances spoken by the same voice - is provided primarily by a similarity shared by the voiced and, therefore, periodic vowel sounds (e.g., a common fundamental and formant structure), whereas unvoiced consonants constitute the noisy, aperiodic onsets and offsets of these periodic sounds (Bregman, 1990). Correspondingly, differences between successive vowels (e.g., /a/ and /e/; induced by different vocal tract shapes) superimposed on this common ground are likely to yield strong serial order cues and, therefore, are more likely to disrupt serial recall than are changes between consonants. ${ }^{2}$

The notions that (1) vowel changes play a critical role in the phonological dissimilarity effect and (2) this is because of the greater propensity for vowel changes (as opposed to consonant changes) to yield serial order information gain support from studies in which serial recall has been examined for items differing only in terms of their consonants, as compared with vowels. Specifically, several studies have shown that vowel-only-changing items (e.g., /bæb/, /bıb/, /bcb/, /bub/ . . ) are far more easily recalled in order than are consonant-only-changing items (/bæb/, /dæb/, /gæb/, /kæb/ . .; e.g., Cole, 1973; Surprenant \& Neath, 1996). Thus, a reasonable, albeit indirect, inference that can be made here is that the ease with which items can be recalled in order when they are attended seems to predict their disruptive potency when they are used as irrelevant sound (see Macken, Phelps, \& Jones, 2005).

Moreover, a sequence of changing vowels may have been particularly likely to compete for the control of action (Hughes \& Jones, 2005; Neumann, 1996) and to interfere with the process of seriating the to-be-remembered items, because verbal serial memory is itself, as was noted above, heavily reliant on changing vowel information. However, although interference stemming from the conflicting vowel information contained within each sequence may have contributed to the present effects (see Hughes \& Jones, 2005), we think that it is unlikely that the effect can be explained completely by such conflict; changing-state effects have been obtained in the absence of any similarity (in terms of vowel information or otherwise) between the content of the relevant and the irrelevant sequences (e.g., Jones, Farrand, Stuart, \& Morris, 1995; Jones \& Macken, 1993). However, a future study could test this more directly by examining whether or not vowel-changing irrelevant sequences are more disruptive than consonant-changing irrelevant sequences in the absence of any vowel-changing information in the to-be-remembered sequence.

In conclusion, we have shown that not all attributes of speech are equally potent in terms of their power to induce a within-stream phonological dissimilarity (or changing- 
state irrelevant speech) effect. The vowel-changing-state effect can be explained by recourse to an interference-byprocess account, whereby only a sequence of changing elements that share a common ground will yield salient serial order information, which in turn conflicts with the process of seriating the to-be-remembered material.

\section{REFERENCES}

Baddeley, A. D. (2000). The phonological loop and the irrelevant speech effect: Some comments on Neath (2000). Psychonomic Bulletin \& Review, 7, 544-549.

Bregman, A. S. (1990). Auditory scene analysis: The perceptual organization of sound. Cambridge, MA: MIT Press.

Bridges, A. M., \& Jones, D. M. (1996). Word dose in the disruption of serial recall by irrelevant speech: Phonological similarity or changing state? Quarterly Journal of Experimental Psychology, 49A, 919-939.

Buchner, A., Irmen, L., \& ERdfelder, E. (1996). On the irrelevance of semantic information for the "irrelevant speech" effect. Quarterly Journal of Experimental Psychology, 49A, 765-779.

Cole, R. A. (1973). Different memory functions for consonants and vowels. Cognitive Psychology, 4, 39-54.

Colle, H. A. (1980). Auditory encoding in visual short-term recall: Effects of noise intensity and spatial location. Journal of Verbal Learning \& Verbal Behavior, 19, 722-735.

Colle, H. A., \& Welsh, A. (1976). Acoustic masking in primary memory. Journal of Verbal Learning \& Verbal Behavior, 15, 17-31.

Hughes, R. W., \& Jones, D. M. (2005). The impact of order incongruence between a task-irrelevant auditory sequence and a task-relevant visual sequence. Journal of Experimental Psychology: Human Perception \& Performance, 31, 316-327.

Jones, D. M., Alford, D., Bridges, A., Tremblay, S., \& Macken, W. J. (1999). Organizational factors in selective attention: The interplay of acoustic distinctiveness and auditory streaming in the irrelevant sound effect. Journal of Experimental Psychology: Learning, Memory, \& Cognition, 25, 464-473.

Jones, D. M., Farrand, P., Stuart, G., \& Morris, N. (1995). Functional equivalence of verbal and spatial information in serial shortterm memory. Journal of Experimental Psychology: Learning, Memory, \& Cognition, 21, 1008-1018.

Jones, D. M., \& MACKEN, W. J. (1993). Irrelevant tones produce an irrelevant speech effect: Implications for phonological coding in working memory. Journal of Experimental Psychology: Learning, Memory, \& Cognition, 19, 369-381.

Jones, D. M., \& MACKEN, W. J. (1995a). Organizational factors in the effect of irrelevant speech: The role of spatial location and timing. Memory \& Cognition, 23, 192-200.

Jones, D. M., \& Macken, W. J. (1995b). Phonological similarity in the irrelevant speech effect: Within- or between-stream similarity? Journal of Experimental Psychology: Learning, Memory, \& Cognition, 21, 103-115.

Jones, D. M., Madden, C., \& Miles, C. (1992). Privileged access by irrelevant speech to short-term memory: The role of changing state. Quarterly Journal of Experimental Psychology, 44A, 645-669.

Jones, D. M., Saint-Aubin, J., \& Tremblay, S. (1999). Modulation of the irrelevant sound effect by organizational factors: Further evidence from streaming by location. Quarterly Journal of Experimental Psychology, 52A, 545-554.

Jones, D. M., \& Tremblay, S. (2000). Interference by process or context? A reply to Neath (2000). Psychonomic Bulletin \& Review, 12, 550-558.

Jusczyk, P. W., Goodman, M. B., \& Baumann, A. (1999). Ninemonth-olds' attention to sound similarities in syllables. Journal of Memory \& Language, 40, 62-82.

Larsen, J. D., Baddeley, A. D., \& Andrade, J. (2000). Phonological similarity and the irrelevant speech effect: Implications for models of short-term verbal memory. Memory, 8, 145-157.

LeCompte, D. C. (1995). An irrelevant speech effect with repeated and continuous background speech. Psychonomic Bulletin \& Review, 2, 391-397.
LeCompte, D. C. (1996). Irrelevant speech, serial rehearsal and temporal distinctiveness: A new approach to the irrelevant speech effect. Journal of Experimental Psychology: Learning, Memory, \& Cognition, 22, 1154-1165.

LeCompte, D. C., \& Shaibe, D. M. (1997). On the irrelevance of phonological similarity to the irrelevant speech effect. Quarterly Journal of Experimental Psychology, 50A, 100-118.

Macken, W. J., Phelps, F., \& Jones, D. M. (2005). What causes auditory distraction? Manuscript in preparation.

Miles, C., Jones, D. M., \& Madden, C. A. (1991). Locus of the irrelevant speech effect in short-term memory. Journal of Experimental Psychology: Learning, Memory, \& Cognition, 17, 578-584.

NEATH, I. (2000). Modeling the effects of irrelevant speech on memory. Psychonomic Bulletin \& Review, 7, 403-423.

Neumann, O. (1996). Theories of attention. In O. Neumann \& A. F. Sanders (Eds.), Handbook of perception and action (Vol. 3, pp. 389446). London: Academic Press.

Salamé, P., \& BadDEley, A. D. (1982). Disruption of short-term memory by unattended speech: Implications for the structure of working memory. Journal of Verbal Learning \& Verbal Behavior, 21, 150-164.

Surprenant, A. M., \& Neath, I. (1996). The relation between discriminability and memory for vowels, consonants, and silent-center vowels. Memory \& Cognition, 24, 356-366.

Tremblay, S., \& Jones, D. M. (1999). Change of intensity fails to produce an irrelevant sound effect: Implications for the representations of unattended sound. Journal of Experimental Psychology: Human Perception \& Performance, 25, 1005-1015.

\section{NOTES}

1. Strictly speaking, therefore, the term phonological dissimilarity effect is a misnomer, and acoustic dissimilarity effect would be more appropriate. That is, according to the changing-state hypothesis, it is not the similarity between the irrelevant speech tokens at an abstract, speech-based, phonological level of analysis that mediates the disruption but, rather, dissimilarity at a precategorical, acoustic level. Nonetheless, to be in keeping with previous articles on this specific topic we will retain the term phonological dissimilarity effect.

2. Although it could be argued that a consonant-only-changing sequence has a common ground provided by the steady-state vowel information within it, our view is that the change (that mediates the disruption) must occur on the same attribute of the sequence as that which provides the common ground - that is, the vowels must themselves be changing.

\begin{tabular}{|c|c|}
\hline \multicolumn{2}{|c|}{$\begin{array}{l}\text { APPENDIX } \\
\text { Stimuli Used for the Final-Consonant-Changing and } \\
\text { All-Components-Changing Conditions in Experiment } 1\end{array}$} \\
\hline $\begin{array}{l}\text { Final Consonant } \\
\text { Changing }\end{array}$ & $\begin{array}{l}\text { All Components } \\
\text { Changing }\end{array}$ \\
\hline$[\mathrm{fct}]$ & [vik] \\
\hline$[\mathrm{fem}]$ & [fuv] \\
\hline$\left[\mathrm{fed}_{3}\right]$ & [geydz] \\
\hline [fen] & [zuk] \\
\hline$[\mathrm{f} \varepsilon \mathrm{g}]$ & [fat] \\
\hline [feb] & [zut] \\
\hline$[\mathrm{f} \varepsilon \theta]$ & [v $\wedge \mathrm{p}]$ \\
\hline$\left[\mathrm{f} \varepsilon \int\right]$ & [zob] \\
\hline [fez] & {$\left[\mathrm{dot} \int\right]$} \\
\hline [fev] & [dzoyf $]$ \\
\hline [fes] & [fId3] \\
\hline [fek] & [dzays] \\
\hline
\end{tabular}

Note-Taken from Jusczyk, Goodman, and Baumann (1999).

(Manuscript received July 1, 2004;

revision accepted for publication January 15, 2005.) 